\title{
CONNECTION BETWEEN THE CME VELOCITIES AND DECAMETER RADIO BURSTS PARAMETERS FROM URAN-4 OBSERVATIONS
}

\author{
Galanin V.V. ${ }^{1}$, Isaeva E.A. ${ }^{1,2}$, Kravetz R.O. ${ }^{1}$ \\ ${ }^{1}$ Observatory URAN-4 of the Institute of Radio Astronomy of the National Academy of \\ Sciences of Ukraine,gvv@breezein.net, krro@ukr.net \\ ${ }^{2}$ Research Institute "Astronomy Observatory of Odessa I.I. Mechnikov National \\ University”isaeva-ln@mail.ru
}

ABSTRACT. The paper reports the results of the research of connection between the coronal mass ejections (CME) with the IV type continual decameter bursts parameters. As the parameters characterizing the CME velocity, we used the integrated flux of the radio bursts and background intensity on 20 and $25 \mathrm{MHz}$ frequencies. The analysis demonstrated that the connection between the CME velocity and IV type bursts increases, if we take into account the intensity of the radio bursts and background on two polarizations at a given frequency. In this case, the correlation coefficient is $\approx 0.75$.

Keywords: coronal mass ejection, radio burst.

\section{Introduction}

The most simple and accessible way for diagnostics of the coronal mass ejection (CME) parameters is using the parameters of the radio bursts. It is well known that the vast majority of metric and hectometric bursts of IV and II type are connected with the CME (Tsap et al., 2013). This paper demonstrates that there is a sufficiently strong connection between the CME velocity and integral flux of the microwave bursts, however the connection between CME velocity and parameters of metric and decametric bursts of II type proved to be low.

\section{Basic data and processing results}

The measurements were carried out by decametric radio telescope Uran-4 (PT) (Galanin et al., 1989). Radio telescope URAN-4 is a part of the very long base radio interferometer system (VLBI), which is located on the territory of Ukraine from west to east. The operating range of the instrument is $10-30 \mathrm{MHz}$.

Radio telescope antenna is represents the electrically controlled phased array with linear size 232.5 x $22.5 \mathrm{~m}$. and consist of 128 crossed dipoles. It enables to select two polarization components of the signal. Width of the instrument antenna direction diagram at the level of halfpower on $25 \mathrm{MHz}$ frequency is 2.7 X 22 degrees. In VLBI mode of the interferometer, the resolution of 2 seconds is provided.

After the modernization of equipment in 2011, the observations were initiated on 20 and $25 \mathrm{MHz}$ frequencies. For our measurements, we used the method of "translucence" of the outer regions of the corona by radiation of discrete radio sources. This method allowed us to obtain information about the environment of radio waves propagation and to measure the intensity of the solar radio radiation.

Figure 1 demonstrates an example of recording on two polarizations on 20 and $25 \mathrm{MHz}$ frequencies of the continual burst fixed by RT Uran-4 on June 27.2012. Fig. 2 provides a record of the same burst fixed by SVTO observatory.

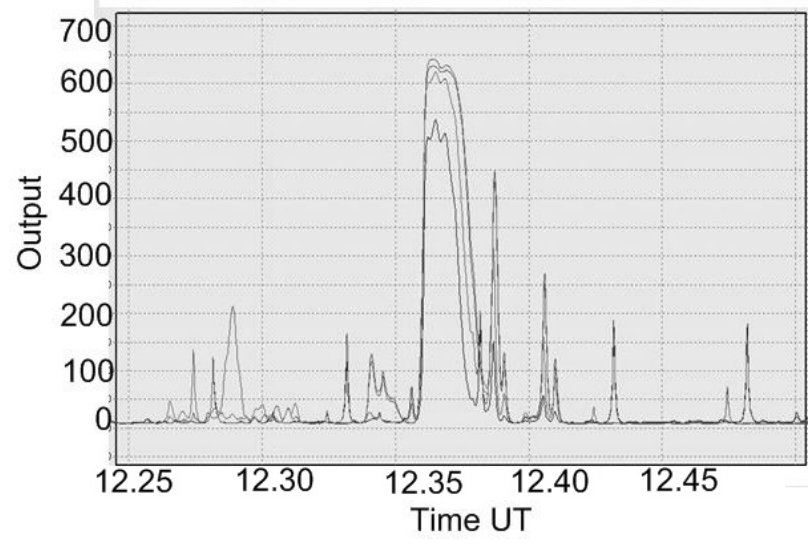

Figure 1: Record of the continual solar burst recorded on 27.06.2012 by radio telescope URAN-4 on two polarizations on 20 and $25 \mathrm{MHz}$. frequencies.

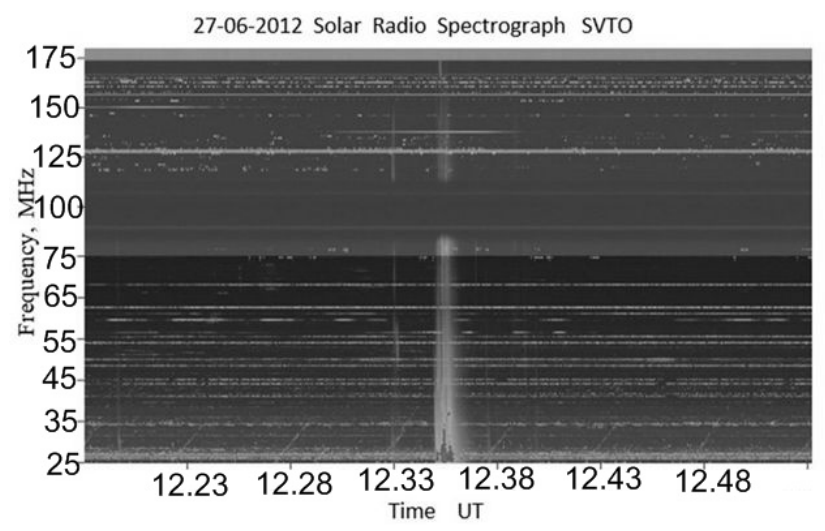

Figure 2: Observation on spectrograph of SVTO observatory of the continual burst connected with CME, 27.06.2012. 
In this paper we restrict ourselves to the research of the connection between CME velocity $V_{C M E}$ and parameters of the IV type continual decametric bursts obtained from measurements by the radio telescope URAN-4. We selected the CME velocity from catalog SOHO / LASCO. We analyzed the connection between the CME velocity with such parameters as the duration, maximum and integral value of the burst flux for two polarizations, as well as the value of background.

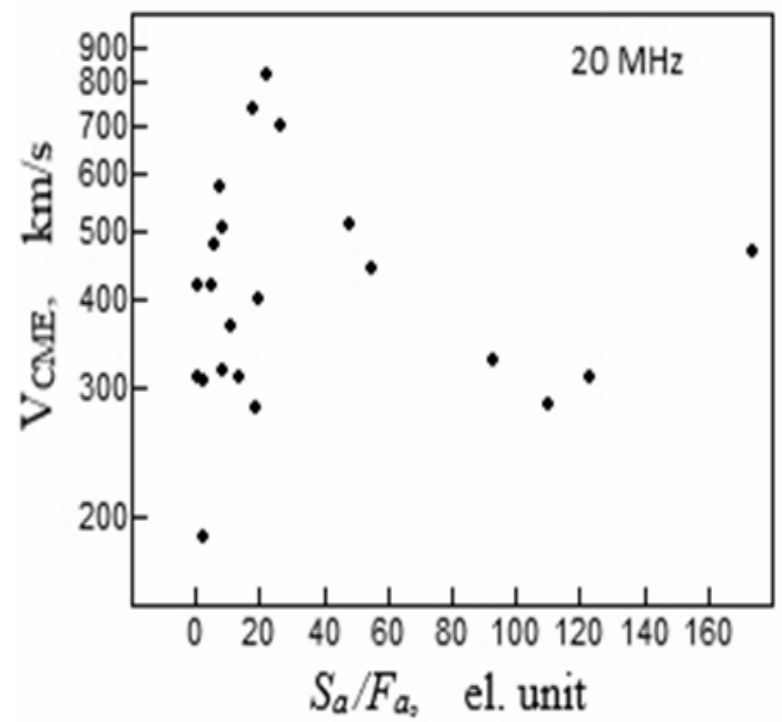

a)

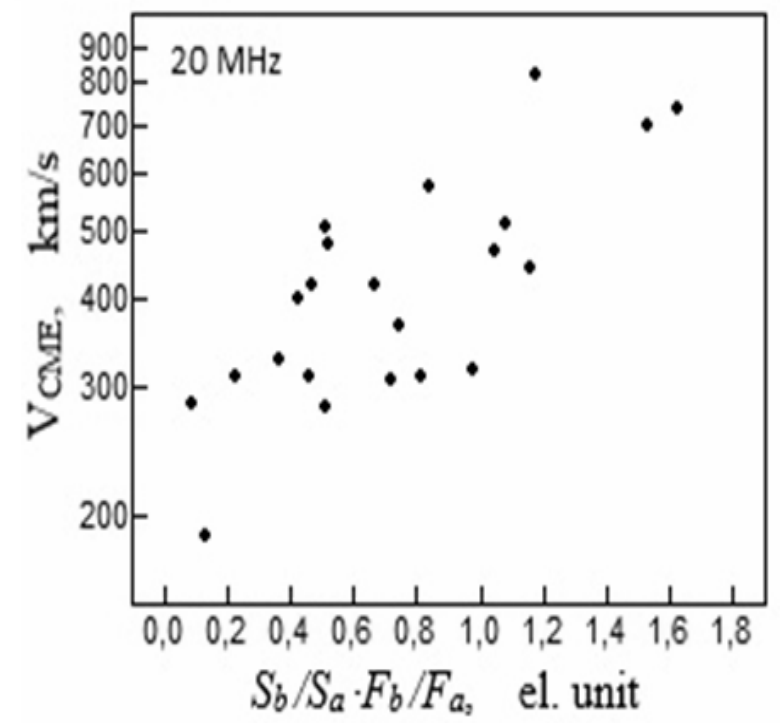

b)

Figure 3: a) Correlation coefficient $r$ between $V C M E$ and $\mathrm{Sa} / \mathrm{Fa}, \quad r \sim 0.06$; b) Correlation coefficient $r$ between $V C M E$ and $S b / S a * F b / F a, r \sim 0.75$.
Comparative analysis demonstrated that the connection between the CME ejection and each listed parameter proved to be low, which is quite consistent with previous results (Tsap et al., 2013).. Fig. 3 a) shows an example of the research of CME velocity and integral flux of burst $S_{a}$ normalized to the value of background $F_{a}$. In this case, the connection between $V_{C M E}$ and $S_{a} / F_{a}$ is absent. However, the connection between the CME velocity and the parameters of the radio bursts significantly increases if values of the burst flux and background on two polarizations are used simultaneously (1), see. Fig. 3b).

$$
\begin{gathered}
\operatorname{Lg} V_{C M E}=-0.0002 \cdot S_{a}-0.02220 \cdot F_{a}+0.0002 \cdot S_{b}+ \\
0.0539 \cdot F_{b} \sim S b / S a * F b / F a
\end{gathered}
$$

where $S_{a}, F_{a}$ and $S_{b}, F_{b}$ - integral flux of the burst and value of the background on two polarizations $a$ and $b$, respectively.

From correlation (1) it is seen how the CME velocity is connected with the parameters $\mathrm{S}_{\mathrm{a}}, \mathrm{F}_{\mathrm{a}}$ and $\mathrm{S}_{\mathrm{b}}, \mathrm{F}_{\mathrm{b}}$. Specifically, the ratio between the flux of burst and ratio of the background on two polarizations proved to be of fundamental importance. Unfortunately, such selection includes only 22 events, which didn't enable to get a true dependence of the $\mathrm{CME}$ velocity on radio burst parameters due to the small amount of data.

\section{Conclusions}

1. The results of implemented researches indicate that the CME velocity is connected to some extent with the polarization characteristics of IV type decametric radio bursts.

2. Further research in this direction is necessary.

\section{References}

Tsap Yu.T. et al.: 2013, J.CrAO, 109, 4, 121.

Galanin V.V. et al.: 1989, Cinematics and Physics of Celestial Bodies, 5, 5, 87-90. 